\title{
Interval Debulking Surgery in Ovarian Cancer
}

\author{
Meeta Singh', Rajshree Jha', Samjhana Dhakal' \\ Dept of Obs / Gyn, TUTH, Institute of Medicine', \\ Paropakar Maternity and Womens Hosp², \\ Kathmandu, Nepal ${ }^{2}$
}

\begin{abstract}
Primary debulking surgery's (PDS) role in ovarian cancer there is no dispute, as the currently accepted management of treatment of advanced stage ovarian cancer is PDS in order to achieve an optimal cytoreduction as the amount of residual tumour is one of the most important prognostic factors for survival of women with epithelial ovarian cancer. Several authors have reported PDS is associated with high mortality and morbidity rates, when an optimal surgical procedure, defined as a removal of tumours to $<1$ to $2 \mathrm{~cm}$ in size, required for advanced stage disease (III to IV). So interval debulking sugery (IDS) should be performed in unresectable cases or in the advanced ovarian cancers. However the role of IDS is still not well defined, at present where PDS is feasible and permits a minimal residual tumour to achieve an optimal cytoreduction with an acceptable morbidity, it should be performed in patients with stage II up to IIIC disease with minor carcinomatosis. IDS should be performed in the case of patients with massive unresectable cases. The preliminary results so far reported should be confirmed by a randomized study or levellevidence being conducted by the EORTC (European Organisation for Research and Treatment of Cancer) group to reinforce the randomized study of Maria et al before recommending IDS in cancer of ovary
\end{abstract}

Keywords: IDS, PDS, unrespectable ovarian cancer

\section{Introduction}

Primary ovarian cancer surgery is performed to achieve optimal cytoreduction as the amount of residual tumour is one of the most important prognostic factors for survival of women with epithelial ovarian cancer ${ }^{1-3}$. The currently accepted management of treatment of advanced stage ovarian cancer is a primary debulking surgery (PDS) in order to achieve an optimal cytoreduction (defined as residual tumour $<2 \mathrm{~cm}$ ) followed by curative therapy ${ }^{1-6}$. However, cytoreductive surgery is associated with high mortality and morbidity rates, and with misleading results on survival ${ }^{4,7-11}$. Such results suggest that this surgical strategy should be reconsidered; particularly in patients with massive intra-abdominal spread several non-randomized studies have discussed the benefit of induction chemotherapy in such patients ${ }^{11-19}$. As PDS is associated with high mortality and morbidity rates, over the past few years interval debulking surgery (IDS) that is debulking surgery after induction chemotherapy in inoperable cases, particularly in patients with massive intra-abdominal spread is the surgical strategy. Although the value of primary cytoreductive surgery for epithelial ovarian cancer is beyond doubt, the value of debulking surgery after induction chemotherapy has not yet been defined.

Inoperable means to some team who are doing research as 'unresectable' peritoneal involvement (involvement of hepatic pedicle and/or mesentery and/or para-aortic involvement above the level of the left renal vessels and/or involvement of the diaphragmatic muscle or in the case of patients for whom surgical resection is feasible but with multiple bowel resection or extensive surgery (involvement of at least two segments of the digestive tract, and/or involvement of the splenic hilus and pancreas) and therefore with a high risk of postoperative morbidity. While to some teams, the

\section{Correspondence}

Dr. Meeta Singh MD

Prof. \& Head, Dept of Obs / Gyn, TU Teaching Hosp

Institute of Medicine,

Phone : 9841611699

Email : singhmita@hotmail.com 
selection of 'unresectable' tumours is performed using preoperative abdomino pelvic computed tomography (CT), ${ }^{15,16}$ While Sangili et al did a study to investigate the predictive value of serum CA-125 levels to ability of PDS in patients with advanced epithelial ovarian carcinoma $^{20}$.

The value of cytoreductive surgery in the management of ovarian cancer has been debated for years. The reasons for cytoreductive surgery are manifold. Large tumors with relatively poor central blood supplies and the areas with the lowest growth rates are both rather insensitive to cytotoxic drugs ${ }^{21}$. In better-perfused small, residual tumors, the growth rate and the diffusion of chemotherapeutic agents are higher, factors that are apt to increase the efficacy of chemotherapy. The removal of large tumors also reduces the likelihood that drug-resistant clones will appear as a result of spontaneous mutations ${ }^{22}$. Moreover, small tumors require fewer cycles of chemotherapy, thus decreasing the probability of drug-induced resistance. Some studies reported significantly longer media survival of patients who had IDS after chemotherapy than of those who had conventional treatment of primary surgery and adjuvant chemotherapy ${ }^{10,18}$. While several studies have discussed the benefit of IDS in inoperable cases, particularly in patients with massive intra-abdominal spread. Several studies of 23-25 showed improved survival of patients with residual tumors less than 1 $\mathrm{cm}$ in diameter after primary surgery, as compared with patients with larger lesions ${ }^{4,6}$. Various studies have discussed the benefit of induction chemotherapy in such patients $^{10-19}$. In a case-control study of patients with minimal residual disease, Eisenkop et al. reported that patients whose small lesions were all resected survived significantly longer than patients in whom such lesions were not resected.(6) An exposed-non-exposed study including a group of 34 patients who underwent an IDS and were matched to control group of 34 patients treated with PDS in a study of P Morace et al (26) found that IDS in patients with 'unresectable'advanced stage ovarian cancer seems to offer the same chance of survival as PDS, but with a reduction of morbidity and hospital stay, and improvement in the quality of life of patients.

The study demonstrate that survival of patients treated for advanced stage 'unresectable' ovarian cancer with IDS is similar to patients treated with PDS, but with a reduced morbidity and a better quality of life (reduction of enterostomy rates). While Vergote and Kuhn(10, 18), reported significantly longer median survival of patients who had IDS after chemotherapy than of those who had conventional treatment of primary surgery and adjuvant chemotherapy Similarly van der Burg (27) showed significantly longer survival in the IDS group which was still present after a 10 -year follow- up. Likewise Chan and Rose $(28,29)$ found beneficial effect in survival after IDS

On the other hand, there are various studies who do not agree to the beneficial effect of IDS like Hoskins et al and Hacker et al.(3) and Hoskins et al.(4) reported that despite optimal cytoreduction, the survival of patients with large intra abdominal metastases before resection was significantly worse than that of patients with small initial intra abdominal lesions. These studies suggest that in addition to residual disease after cytoreduction, intrinsic tumor factors are of prognostic importance. They also raise the question of whether cytoreduction has a significant effect on survival among patients with the same size tumors and the same intrinsic prognostic factors. Netjer et al however, reported just the opposite, 23 Patients with optimal cytoreduction at intervention surgery had poorer rates of survival than patients with optimal cytoreduction at primary surgery ${ }^{30}$. Moreover, the survival of patients with optimal cytoreduction at intervention surgery was the same as that of patients with suboptimal cytoreduction. All these studies, in favor or not in favor of IDS are mainly non randomized studies and include only a small no of cases.

However the randomized study done by Maria et al for the Gynecological Cancer Cooperative Group of the European Organization for Research and Treatment of Cancer (EORTC) From March 1987 to May 1993, 425 patients were enrolled in the study ${ }^{31}$. Eligible patients had to have biopsy-proved epithelial ovarian carcinoma with an International Federation of Gynecology and Obstetrics stage of IIb to IV, (32 new) they had to have undergone primary surgery no more than six weeks before treatment began. Clinical response was assessed according to the standard WHO response criteria $^{33}$. A complete response was defined pathologically as the absence of macroscopic and microscopic tumor at surgery. Optimal cytoreduction was defined as the reduction of all tumor lesions to less than $1 \mathrm{~cm}$ in diameter.

All randomized patients with some follow-up information were included in the analyses of survival and progression-free survival, which were performed strictly according to the intention-to-treat principle. Survival and progression-free survival curves were calculated for each treatment group with Kaplan-Meier estimates and compared with the log-rank test $\mathrm{t}^{34,35}$. The median progression-free survival was 18 months for the patients who had debulking surgery and 13 months for the patients who did not have debulking surgery. The percentage of patients alive and free of progressive disease at two years was 38 percent in the former group and 26 percent in the latter. The disease progressed in 8 percent of the patients who underwent debulking surgery and 12 percent of those who did not. 
Both overall survival and progression-free survival were significantly longer $(\mathrm{P}=0.01)$ for the patients who underwent debulking surgery. The difference in survival was more substantial when patients with stage IV disease were excluded ( $\mathrm{P}=0.003$ for overall survival and $\mathrm{P}=0.002$ for progression-free survival). The median survival was 26 months for the patients who underwent debulking surgery and 20 months for those who did not. The proportion of patients alive at two years was 56 percent in the former group and 46 percent in the latter group. Overall, surgery reduced the unadjusted risk of death by 31 percent (the hazard rate, or the risk of death at any given moment). Patients whose lesions measured less than $1 \mathrm{~cm}$ before cytoreduction survived significantly longer (median, 41.6 months; $\mathrm{P}<0.001$ ) than patients whose lesions measured more than $1 \mathrm{~cm}$ after debulking surgery (median survival, 19.4 months); survival in the latter group was similar to overall survival in the group that did not undergo debulking surgery (median survival, 20.0 months). Patients with optimal cytoreduction (whose remaining lesions measured less than $1 \mathrm{~cm}$ ) also survived significantly longer (median survival, 26.6 months; $\mathrm{P}=0.04$ ) than patients with suboptimal cytoreduction. Likewise, the progression-free survival of patients with optimal cytoreduction was significantly longer (median survival, 23.3 months; $\mathrm{P}=0.003$ ) than the progression-free survival of patients with suboptimal cytoreduction.

\section{Conclusion}

PDS's role in ovarian cancer there is no dispute but IDS is still not well defined, at present where PDS is feasible and permits a minimal residual tumour with an acceptable morbidity to be obtained; it should be performed in patients with stage II up to IIIC disease with minor carcinomatosis. IDS should be performed in the case of patients with massive unresectable cases. The preliminary results so far reported should be confirmed by a randomized study or level levidence being conducted by the EORTC (European Organisation for Research and Treatment of Cancer) group to reinforce the randomized study of Marira etal before recommending IDS in cancer of ovary.

\section{References}

1. Griffiths TC, Parker LM, Fuller AF. Role of cytoreductive surgical treatment in the management of advanced ovarian cancer. Cancer Treat Rep 1979; 63: 235-240.

2. Bristow RE, Tomacruz RS, Armstrong DK, Trimble EL, Montz FJ. Survival effect of maximal cytoreductive surgery for advanced ovarian carcinoma during the platinum era: a meta-analysis. Journal of Clinical Oncology 2002; 20:1248-59.
3. Hoskins WJ, McGuire WP, Brady MF, et al. The effect of the diameter of largest residual disease on survival after primary cytoreductive surgery in patients with suboptimal residual epithelial ovarian carcinoma. Am J Obstet Gynecol 1994; 170: 974-980.

4. Michel G, De Iaco P, Castaigne D, et al. Extensive cytoreductive surgery in advanced ovarian carcinoma. Eur J Gynaec Oncol 1997; 18: 9-15.

5. Hacker NF, Berek JS, Lagasse LD, et al. Primary cytoreductive surgery for epithelial ovarian cancer. Obstet Gynecol 1983; 61: 413-420.

6. Eisenkop SM, Friedman RL, Wang HJ. Complete cytoreductive surgery is feasible and maximizes survival in patients with advanced epithelial ovarian cancer: a prospective study. Gynecol Oncol 1998; 69: 103-108.

7. Gadducci A, Iacconi P, Cosio S, Fanucchi A, Cristofani R, Genazzani R. Complete salvage surgical cytoreduction improves further survival ofpatients with late recurrent ovarian cancer. Gynecol Oncol 2000; 79:344-9.

8. Vaccarello L, Rubin SC, Vlamis V, Wong G, Jones WB, Lewis JL, et al. Cytoreductive surgery in ovarian carcinoma patients with a documentedpreviously complete surgical response. Gynecol Oncol 1995; 57:61-5.

9. Guidozzi F, Ball JH. Extensive primary cytoreductive surgery for advanced epithelial ovarian cancer. Gynecol Oncol 1994; 53: 326330 .

10. Vergote IB, De Wever I, Decloedt J, et al. Neoadjuvant chemotherapy or primary debulking surgery in advanced ovarian carcinoma: a retrospective analysis of 285 patients. Gynecol Oncol 1998; 71: 431-436.

11. Lawton FG, Redman CW, Luesley DM, et al. Neoadjuvant (cytoreductive) chemotherapy combined with intervention debulking surgery in advanced epithelial ovarian cancer. Obstet Gynecol 1989; 73: 61-65.

12. Chambers JT, Chambers SK, Voynick IM, Schwartz PE. Neoadjuvant chemotherapy in stage X ovarian carcinoma. Gynecol Oncol 1990; 37: 327-331.

13. Jacob JH, Gershenson DM, Morris M, et al. Neoadjuvant chemotherapy and interval debulking for advanced epithelial ovarian cancer. Gynecol Oncol 1991; 42: 146-150.

14. Lim JTW, Green JA. Neoadjuvant carboplatin and ifisfamide chemotherapy in inoperable FIGO stage III and IV ovarian carcinoma. Clin Oncol 1993; 5: 198-202.

15. Surwit E, Childers J, Atlas I, et al. Neoadjuvant chemotherapy for advanced ovarian cancer. Int J Gynecol Cancer 1996; 6: 356-361.

16. Schwartz PE, Rutherford TJ, Chambers JT, et 
al. Neoadjuvant chemotherapy for advanced ovarian cancer: Long-term survival. Gynecol Oncol 1999; 72: 93-99.

17. Kayikcioglu LF, Kose MF, Boran N, et al. Neoadjuvant chemotherapy or primary surgery in advanced epithelial ovarian carcinoma. Int $\mathrm{J}$ Gynecol Cancer 2001; 11: 466-470.

18. Kuhn W, Rutke S, Spathe K, et al. Neoadjuvant chemotherapy followed by tumor debulking prolongs survival for patients with poor prognosis in International Federation of Gynecology and Obstetrics Stage IIIC ovarian carcinoma. Cancer 2001; 92: 2585-2591.

19. Ansquer $\mathrm{Y}$, Leblanc E, Clough $\mathrm{K}$, et al. Neoadjuvant chemotherapy for unresectable ovarian carcinoma. Cancer 2001; 1: 2329-2334.

20. Saygili U.; Guclu S.; Uslu T.; Erten O.; Demir N.; Onvural A. Optimal Primary Cytoreduction in patients with Advanced Ovarian Carcinoma. Gynecologic Oncology, Volume 86, Number 1, July 2002, pp. 57-61(5)

21. Skipper H E. Thoughts on cancer chemotherapy and combination modality therapy. JAMA 1974; 230:1033-1035.

22. Goldie JH, Coldman AJ. A mathematic model for relating the drug sensitivity of tumors to their spontaneous mutation rate. Cancer Treat Rep 1979; 63:1727-1733.

23. Vogl SE, Pagano M, Kaplan BH, Greenwald E, Arseneau J, Bennett B. Cis-platin based combination chemotherapy for advanced ovarian cancer: high overall response rate with curative potential only in women with small tumor burdens. Cancer 1983; 51:2024-2030.

24. Redman 1994 \{published data only\} _ Redman CW, Warwick J, Luesley DM, Varma $\bar{R}$, Lawton FG, Blackledge GR. Intervention debulking surgery in advanced epithelial ovarian cancer. British Journal of Obstetrics and Gynaecology 1994; 101:142-6.

25. Omura GA, Bundy BN, Berek JS, Curry S, Delgado G, Mortel R. Randomized trial of cyclophosphamide plus cisplatin with or without doxorubicin in ovarian carcinoma: a Gynecologic Oncology Group study. J Clin Oncol 1989; 7:457-465.
26. Morice P, Brehier-Ollive D, Rey A, Atallah D, Lhomme C, Pautier P, Pomel C, Camatte S, Duvillard P, Castaigne D. (2003). Results of interval debulking surgery in advanced stage ovarian cancer: an exposed-non-exposed study. Ann Oncol 14: 74-77

27. Van Der Burg 2003 van der Burg ME, Vergote I, and Gynecological Cancer Group of the EORTC. The role of interval debulking surgery in ovarian cancer. 28 Current Oncology Reports 2003; 5:473-81.

28. Chan YM, Ng TY, Ngan HY, Wong LC. Quality of life in women treated with neoadjuvant chemotherapy for advanced ovarian cancer: a prospective longitudinal study. Gynecologic Oncology 2003;88:9

29. Rose P G, Nerenston S, Brady M F, Clarke Pearson D, Olt G, Rubin S C, et al. Operative morbidity following primary and interval debulking surgery for advanced ovarian cancer

30. Neijt JP, ten Bokkel Huinink WW, Van Der Burg MEL, et al. Long-term survival in ovarian cancer: mature data from the Netherlands Joint Study Group for Ovarian Cancer. Eur J Cancer 1991; 27:1367-1372.

31. Maria EL, Mare Beuse, Anna Kabierska, et al. Interval cytoreduction in ovarian cancer. The New England Journl of Meicine Volume 333:254-255 July 27,2006 Number4

32. Kottmeier H L. Presentation of therapeutic results in carcinoma of the female pelvis: experience of the annual report on the results of treatment in carcinoma of the uterus, vagina, and ovary. Gynecol Oncol 1976; 4:13-19.

33. World Health Organization. WHO handbook for reporting results of cancer treatments. World Health Organization offset publication no. 48. Geneva: World Health

34. Kaplan EL, Meier P. Nonparametric estimation from incomplete observations. J Am Stat Assoc 1958; 53:457-81.

35. Peto R, Pike MC, Armitage P, et al. Design and analysis of randomised clinical trials requiring prolonged observation of each patient. II. Analysis and examples. Br J Cancer 1977; 35:139. 